\title{
Leucopatía reversible de la protuberancia: en paciente con SIDA y tratamiento retroviral
}

\author{
LUIS CARTIER $^{1,2, *}$, JOSÉ MANUEL MATAMALA ${ }^{1}$, ALONSO YÁÑEZ
}

\section{Pontine reversible leucopathy in an AIDS patient associated with highly active antiretroviral therapy (HAART). Report of one case}

Posterior reversible encephalopathy (PRES) is a condition characterized by T2 and FLAIR hyperintensities in magnetic resonance imaging (MRI) studies, localized preferentially in the occipital-parietal white matter regions. Pathological MRI images located in midbrain, pons, medulla and spinal cord, that could be asymptomatic, were recently included in this entity. These images are interpreted as vasogenic edema, which is caused by arterial hypertension or eclampsia, neurotoxicity related to immunosuppressive agents or chemotherapy, among other causes. We report a 25 years old asymptomatic male with AIDS, with normal blood pressure who after initiating highly active antiretroviral therapy (HAART) reported vertigo. The MRI showed a central pontine T2 hyperintensity with diffusion restriction, which was interpreted as a central pontine myelinolysis $(C P M)$, but the lack of motor symptoms made improbable a real demyelination of the pons. The follow-up MRI revealed complete regression of the images. To our knowledge, this case could be the second report of a reversible leucopathy of the pons in a patient with AIDS, were the MRI images also simulated a CPM. This report extends the knowledge around the variability of the pathogenic interpretation of CPM images and their association with HAART.

(Rev Med Chile 2016; 144: 675-679)

Key words: Acquired Immunodeficiency Syndrome; Antiretroviral Therapy, Highly Active; Myelinolysis, Central Pontine; Posterior Leukoencephalopathy Syndrome.

$\mathrm{D}$ esde el advenimiento de la resonancia magnética (RM) el estudio de la substancia blanca en el sistema nervioso central (SNC) se ha hecho más asequible. Ejemplos son el síndrome de leucoencefalopatía posterior reversible (LPR) y la mielinolisis central pontina (MCP). El diagnóstico de LPR está sustentado principalmente en la RM, más que en las manifestaciones clínicas que pueden ser poco expresivas. Clásicamente la LPR se manifiesta por cefalea, vómitos, alteraciones de la visión y/o compromiso de conciencia. Este síndrome se ha relacionado
'Departamento de Ciencias Neurológicas Oriente, Facultad de Medicina, Universidad de Chile,

Santiago, Chile.

${ }^{2}$ Servicio de Neurología, Hospital del Salvador, Santiago, Chile. ${ }^{3}$ Servicio de Neurorradiología, Instituto de Neurocirugía Dr. Asenjo (INCA), Santiago, Chile.

Financiamiento: Sin

financiamiento.

Conflicto de interés: Los autores declaran no presentar conflicto de intereses.

Recibido el 26 de agosto de 2015, aceptado el 17 de marzo de 2016.

Correspondencia a:

Dr. Luis Cartier Rovirosa Avenida Salvador 364,

Providencia.

Teléfono: +56-2-5754000

Icartier@med.uchile.cl. con hipertensión arterial severa, eclampsia y efectos neurotóxicos atribuibles a antibioterapia o tratamientos inmunosupresores. En la RM se identifican imágenes hiperintensas enT2 y FLAIR localizadas en las regiones parieto-occipitales, aunque pueden llegar a ser holohemisféricas y eventualmente comprometer mesencéfalo, protuberancia, cerebelo, o médula espinal ${ }^{1-3}$. Si bien la intimidad patogénica no está clarificada, se entiende como la expresión de un edema vasogénico vinculado a disfunción circulatoria y a un aumento en la permeabilidad de la barrera hematoencefá- 
lica local ${ }^{4}$. Por otra parte, la mielinolisis central pontina se identifica en la RM por imágenes en la parte central de la protuberancia, hipointensas en T1, hiperintensa en T2 y FLAIR, que restringen en la difusión (DWI), en lo clínico ligadas a tetraplejia o tetraparesia y en lo patológico a desmielinización ${ }^{5}$. Esta habilidad de la RM para identificar patologías de la substancia blanca ha permitido diversificar las etiologías de la MCP, que inicialmente sólo eran atribuibles a desnutrición y alcoholismo ${ }^{6}$, posteriormente se pudo vincular a correcciones rápidas del sodio (hiponatremia) ${ }^{7}$, a cáncer y recientemente a trasplantes hepáticos entre otras causas ${ }^{8}$. Si bien la RM ha permitido formular nuevos diagnósticos y confirmar los establecidos, los estereotipos imagenológicos deben contar siempre con el entorno clínico que avale las imágenes, de otra manera estas dejan de tener la validez presumida. Por ello se presenta un paciente portador de SIDA, que en el comienzo de una terapia antiretroviral mal tolerada mostró en la RM una imagen compatible con una MCP, pero en ausencia de síntomas motores y rápida reversibilidad de la imagen evocó una leucopatía reversible de la protuberancia.

\section{Caso clínico}

Hombre de 25 años sin antecedentes de consumo de alcohol ni drogas ilegales. Hace un mes se le había hecho el diagnóstico de SIDA (recuento de CD4 de 113 células/ul y carga viral 220.000 copias/ $\mathrm{mL}$ ). Dos semanas antes de su ingreso al Hospital del Salvador se había iniciado tratamiento ambulatorio con zidovudina, lamivudina, lopinavir y ritonavir que no toleró, presentando al tercer día cefalea, vómitos y sensación de torpeza intelectual, que obligaron a modificar el tratamiento al séptimo día. El primer esquema fue substituido, por Emtricitabina, Tenofovir, Lopinavir y Ritonavir, y se hospitalizó por la necesidad de evaluar la persistencia de cierta inestabilidad postural y vértigo subjetivo que se originaron durante el uso del primer esquema. Ingresó tolerando bien el nuevo tratamiento con signos vitales normales y un examen general que no mostraba alteraciones, salvo la presencia de adenopatías cervicales. El examen neurológico mostró a un paciente orientado, de inteligencia normal y lenguaje conservado. En el examen de pares craneales el único compromiso era un nistagmus horizontal poco relevante con fase rápida a derecha. No presentaba alteraciones de la marcha, la potencia muscular estaba conservada, con reflejos osteotendíneos normales y reflejos plantares flexores, sin compromiso sensitivo, ni cerebeloso. El nistagmus desapareció al día siguiente, aunque persistía sensación vertiginosa con los cambios de postura. Los exámenes de laboratorio mostraron pancitopenia (hematocrito $26,5 \%$, hemoglobina de $9,3 \mathrm{~g} / \mathrm{dl}$, recuento de glóbulos blancos y plaquetas 3.970 ul y $86.800 \mathrm{ul}$ respectivamente) e hipoalbuminemia (albúmina sérica de $26 \mathrm{~g} / \mathrm{L}$ ), sin alteración de las pruebas hepáticas ni renales, glucosa y electrolitos normales. La RM de cerebro realizada al segundo día de su admisión y con el paciente ya asintomático, reveló una hiperintensidad central pontina en secuencia T2 y FLAIR, con restricción a la difusión, sin reforzamiento con el medio de contraste, sugerente de una MCP (Figura 1). El estudio del líquido cefalorraquídeo (LCR) mostró 0,8 células $\mathrm{mm}^{3}$ (100\% mononucleares), proteína de $0,17 \mathrm{~g} / \mathrm{L}$ y glucosa $0,64 \mathrm{~g} / \mathrm{L}$, VDRL negativo y adenosina desaminasa (ADA) de 1,3 UI/L y el cultivo fue normal. El estudio del VIII par no mostró alteraciones. Los potenciales evocados somatosensoriales y motores por estimulación magnética transcraneal fueron normales. Durante su hospitalización se mantuvo el tratamiento instituido y el paciente evolucionó sin contratiempos. La RM cerebral de control al mes reveló una regresión completa de la imagen pontina (Figura 2). Después de 1 año de seguimiento clínico, el paciente ha permanecido neurológicamente asintomático.

\section{Discusión}

La MCP ha sido una complicación descrita excepcionalmente en pacientes portadores de SIDA. Hasta la fecha se habrían comunicado siete casos y el primer correlato fue un hallazgo de autopsia9. Las MCPs presentadas posteriormente están referidas a pacientes que mostraban un SIDA avanzado y donde alguno de estos enfermos asociaban hiponatremia o hipoalbuminemia, condiciones predisponentes para el desarrollo de la $\mathrm{MCP}^{10-13}$. Cinco de ellos fallecieron en la etapa aguda de la mielinolisis, sólo un enfermo mejoró, siendo éste el único que no presentó compromiso motor en asociación a las imágenes interpretadas como $\mathrm{MCP}^{11}$. La excepcional presencia de la MCP 
a

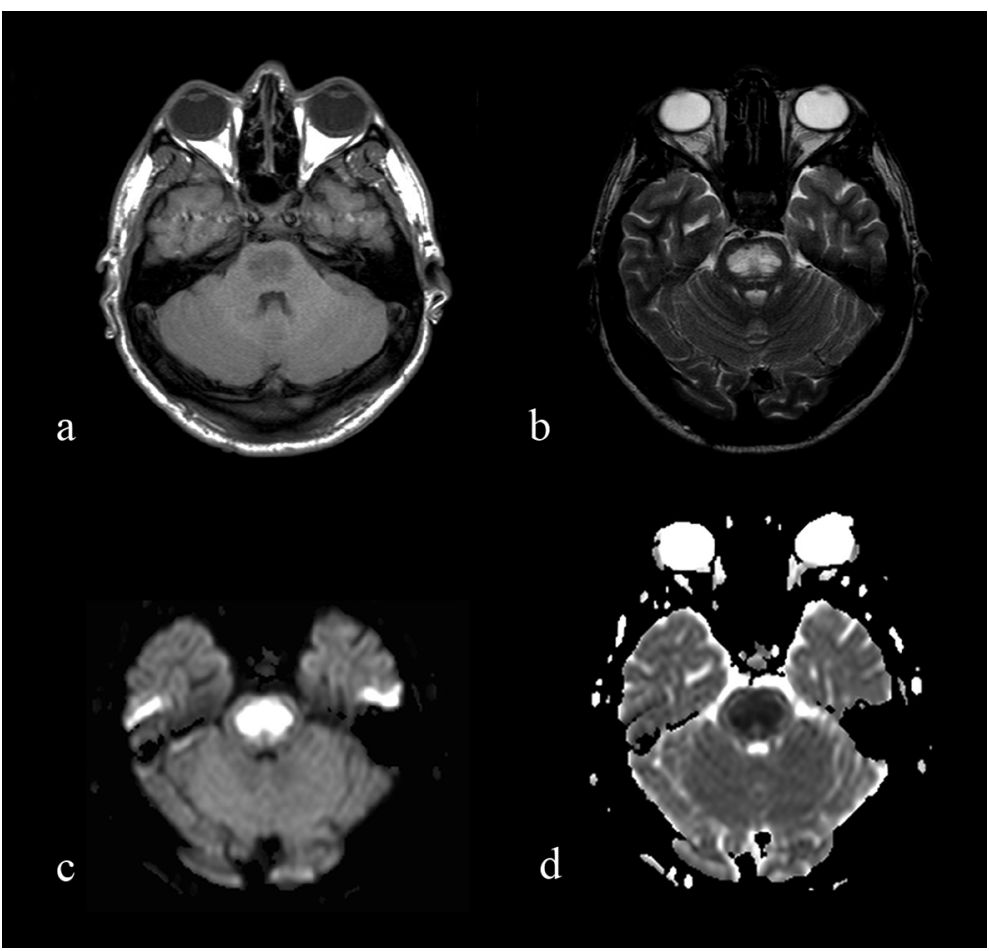

Figura 1. Estudio de RM cerebral cortes axiales de la protuberancia muestran imagen sugerente de CPM en fase aguda que define: en $\mathbf{A}$ hipointensidad de la parte central de la protuberancia (T1), en B hiperintensidad del mismo segmento (T2) en $\mathbf{C}$ hiperintesidad en difusión (DWI) y en $\mathbf{D}$ hipointesidad en el mapa $A D C$.

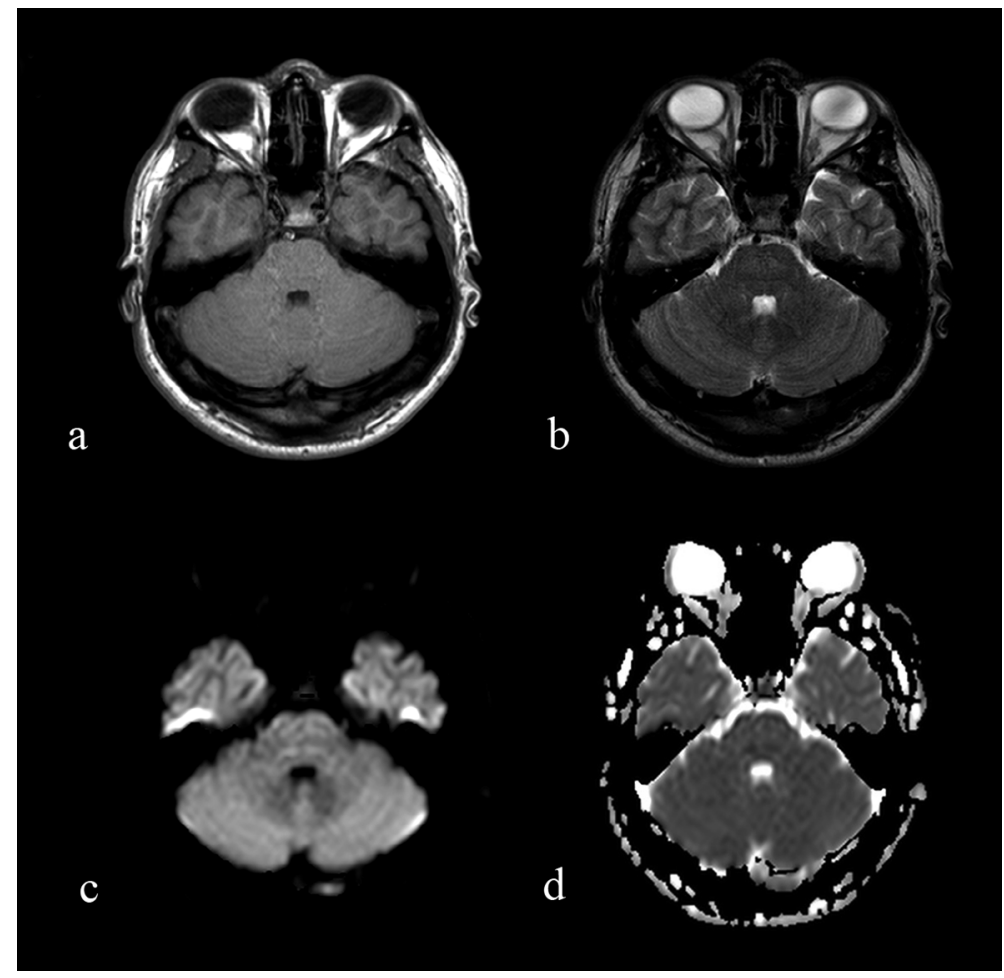

Figura 2. RM cerebral de control de cortes axiales de la protuberancia que muestra regresión completa de las imágenes en $\mathbf{A}$ secuencia T1, en $\mathbf{B}$ secuencia T2, en $\mathbf{C}$ secuencia DWI y en $\mathbf{D}$ el mapa ADC. 
en pacientes con SIDA sugiere que no formaría parte de las complicaciones neurológica propias del VIH, a pesar de la interesante comunicación de Holmes et al, que en base al estudio neuropatológico e inmunohistoquímico de un caso de MCP y SIDA informaron de una inmunorreactividad positiva para p24 en los macrófagos perivasculares encontrados en las lesiones del puente, que para los autores establecía la conexión etiopatogénica entre el VIH y $\mathrm{MCP}^{13}$.

El enfermo que estamos analizando no presentó alteraciones electrolíticas, tampoco cursó con hipertensión arterial y el único antecedente significativo fue su reacción adversa al primer esquema antirretroviral utilizado, cuya intolerancia obligó a descontinuar la zidovudina y la lamivudina. Además obligó a su hospitalización para ser evaluado, debido a la persistencia de una sensación vertiginosa. La RM obtenida al día siguiente de su ingreso, y a dos semanas del comienzo del primer tratamiento retroviral, mostró imágenes compatibles con el diagnostico de una MCP, imágenes que no tenían relación con la condición casi asintomática del paciente. La inexistencia de correlato clínico-imagenológico obligó a desechar la interpretación de las imágenes. Esto basado en la imposibilidad clínica de una desmielinización central de la protuberancia en un paciente que nunca presentó alteraciones motoras. Se pensó en la especial capacidad que tendrían las terapias retrovirales para generar estrés oxidativo o efectos neurotóxicos, que en este caso pudieran haber comprometido la funcionalidad de las neuronas propias del puente, desarrollando alteraciones mitocondriales, así como cambios en la permeabilidad de la barrera hematoencefálica local, debido a los efectos citotóxicos producido por el tratamiento ${ }^{14}$. Sin embargo, el silencio neurológico, el antecedente de una terapia antirretroviral tóxica, su vinculación a imágenes patológicas en la RM y su condición reversible, hicieron surgir como alternativa diagnóstica la leucoencefalopatía posterior reversible, síndrome ampliamente reconocido ${ }^{2}$, donde la experiencia ha demostrado quelas imágenes que acompañan o definen la LPR no están limitadas a regiones occipitoparietales ("posteriores"), sino que pueden expresarse mucho más extensamente ${ }^{15,16} \mathrm{o}$ aparecer aisladamente en un punto del SNC como la protuberancia ${ }^{17}$.

Los pacientes con SIDA no están ajenos a la LPR y entre ellos se han comunicado varios casos de enfermos que mayoritariamente cursaban con una hipertensión arterial severa. La aparición de la LPR no fue atribuida a efectos neurotóxicos originados por el tratamiento, los autores sólo realzaron la acción hipertensiva de los antirretrovirales ${ }^{18,19}$. Sin embargo, Tonioka et al., describieron un paciente con SIDA, no hipertenso y en tratamiento antiretroviral, quien desarrolló una LPR localizado en la protuberancia, una leucopatía reversible de la protuberancia. En este caso la RM inicialmente fue interpretada como MCP y posteriormente reinterpretada como LPR debido a la ausencia de síntomas motores, su reversibilidad ya la ausencia de restricción en la difusión ${ }^{20}$. Ese paciente tiene una definida semejanza con el caso que hemos comunicado, sin embargo, la presencia de restricciones en la difusión que mostró nuestro paciente pareciera hacerlo disímil.

La inexistencia de antecedentes respecto de LPR vinculados al uso de Combivir ${ }^{\circledR}$ (zidovudina más lamivudina), no invalida la excepcional manifestación observada en este paciente. La LPR ha estado comúnmente vinculada con medicamentos que producen efectos sobre la barrera hematoencefálica, condición generalmente idiosincrática, por ello esta leucopatía reversible de la protuberancia podría ser compatible con una terapia antirretroviral, tóxica para este enfermo ${ }^{14}$.

Creemos que este paciente desarrolló una forma de LPR, aunque las restricciones en la difusión pudieran hacerla aparecer como incompatible, sin embargo, la ausencia de síntomas motores como en los casos descritos ${ }^{11,20}$ y la rápida recuperación de las imágenes obligan a sostener que corresponde a una leucopatía reversible de la protuberancia o que estaríamos frente a una nueva entidad clínico-imagenológica.

Nos hemos atrevido a comunicar este interesante y excepcional paciente, buscando extender nuestro conocimiento y activar las inquietudes sobre temas aún incompletamente dilucidados. Así mismo, para incentivarla necesidad de explorar más exhaustivamente los síndromes neurológicos secundarios a intolerancia medicamentosa con estudios de imágenes.

\section{Referencias}

1. Bartynski WS. Posterior reversible encephalopathy syndrome, part 1: Fundamental imaging and clinical features. Am J Neuroradiol 2008; 29: 1036-42. 
2. Lee VH, Wijdicks EF, Manno EM, Rabinstein AA. Clinical spectrum of reversible leukoencephalopathy syndrome. Arch Neurol 2008; 65: 205-10.

3. Mckinney AM, Short J, Truwit CL, Mckinney ZJ, Kozak OS, Santacruz KS, et al. Posterior reversible encephalopathy syndrome: Incidence of atypical regions of involvement and imaging findings. Am J Roentgenol 2007; 189: 904-12.

4. Bartynski WS. Posterior reversible encephalopathy syndrome, part 2: Controversies surrounding pathophysiology of vasogenic edema. Am J Neuroradiol 2008; 29: 1043-9.

5. Lampl C, Yazdi K. Central pontine myelinolysis. Eur Neurol 2002; 47 (1): 3-10.

6. Adams RD, Víctor M, Mancall EL. Central pontinamyelinolysis: a hitherto undescribed disease occurring in alcoholic and malnourished patients. Arch Neurol Psychiatr 1959; 81 (2): 154-72.

7. Dore WD. Osmotic demyelination disorders: central pontine and extrapontinemyelinolysis. Curr Opin Neurol 2000; 13: 691-7.

8. Cartier RL, Armijo MJ, Quiroz ZG, Matamala C JM. Mielinolisis central pontina después del trasplante hepático. Informe de cinco casos. Rev Med Chile 2010; 138: 1264-71.

9. Anders KH, Guerra WF, Tomiyasu U, Verity MA, Vinters HV. The neuropathology of AIDS. UCLA experience and review. Am J Pathol 1986; 124: 537-58.

10. Odero RO, Lacasse A, Farooq A, Cleveland KO. Idiopathic central pontinemyelinolysis in aneunatremic patient with AIDS. AIDS 2009; 23: 1447-9.

11. Maure Noia B, Sopeña B, Argibay Filgueira A, Arias M. Asymptomatic central pontinemyelionolysis and HIV infection. Neurologia 2009; 24:136-7.
12. Katchanov J, Branding G, Stocker H. Central pontinemyelinolysis in advanced HIV infection with tuberculosis and multicentric Castleman's disease. Int J STD AIDS 2013; 24: 583-4.

13. Holmes AH, Esiri M, Morris CS, Edwards A. Central pontinemyelinolysis in a patient with AIDS. J Neurol Neurosurg Psychiatry1992; 55: 631-2.

14. Abers MS, Shandera WX, Kass JS. Neurological and psychiatric adverse effects of antiretroviral drugs. CNS Drugs 2014; 28: 131-45.

15. Havenon A, Joos Z, Longenecker L, Shah L, Ansari S, Digre K, Posterior reversible encephalopathy syndrome with spinal cord involvement. Neurology 2014; 83: 2002-6.

16. Lapuyade B, Sibon I, Jeanin S, Dousset V. Neurological picture: Spinal cord involvement in posteriorreversible encephalopathy syndrome. J Neurol Neurosurg Psychiatry 2009; 80: 35.

17. Abe T, Tokuda Y. Recurrent posterior reversible encephalopathy syndrome of the brainstem in ahypertensive patient with endstagerenal disease. J Emerg Trauma Shock 2014; 7 (3): 242-3.

18. Nightingale S, Wood C, Ainsworth J. The posterior reversible encephalopathy syndrome in HIV infection BMJ Case Reports 2012; doi:10.1136/bcr.01.2012.5647

19. Ridolfo AL, Resta F, Milazzo L, Caramma I, Matacena G, Antinori S, et al. Reversible Posterior Leukoencephalopathy Syndromein 2 HIV-Infected Patients Receiving Antiretroviral Therapy. Brief Report 2008; 46:

20. Tonioka R, Yamamoto Y, Sakai M, Makie T, Mosi M, Uchira $\mathrm{T}$, et al. Convalescence of atypical posterior leukoencephalopathy syndrome in human immunodeficiency virus infection. J Medical Investig 2007; 54: 191-4. 